\title{
Oral and Dental Manifestations of Diamond-Blackfan Anemia: Case Reports
}

\author{
Feyza Otan Ozden ${ }^{\mathrm{a}}$ \\ Kaan Gunduz \\ Bora Ozdenc \\ K. Devrim Iscid \\ Tunc Fisgine
}

\section{ABSTRACT}

Diamond-Blackfan Anemia (DBA) is a red cell aplasia characterized with physical abnormalities. The incidence of the disease is reported to be five to seven (5-7) cases per million births in Europe and 4-5 per million live births in the UK and Netherlands with equal sex ratio. It was first recognized in 1938 but an exact pathophysiology of the disease has not been described yet. These abnormalities are well known, however, detailed oral and dental conditions related with the disease have not been described previously. We herein presented two cases of DBA together with oral and dental findings. Our study is first to report the gingival status of the patients with a complete investigation of any orthodontic or dental abnormalities in these kind of patients. A careful follow up and preventive therapies should not be missed in these kind of patients. (Eur J Dent 2011;5:344-348)

Key words: Diamond-Blackfan Anemia; Dental; Periodontal; Oral.

\section{INTRODUCTION}

Diamond-Blackfan Anemia (DBA) is a chronic, pure erythrocyte aplasia characterized by congen-

a Department of Periodontology, Faculty of Dentistry, Ondokuz Mayis University, Samsun, Turkey.

b Department of Oral Diagnosis and Radiology, Faculty of Dentistry, Ondokuz Mayis University, Samsun, Turkey.

c Department of Oral and Maxillofacial Surgery, Faculty of Dentistry, Ondokuz Mayis University, Samsun, Turkey.

d Department of Orthodontics, Faculty of Dentistry, Ondokuz Mayis University, Samsun, Turkey.

e Department of Pediatric Hematology, Faculty of Medicine, Ondokuz Mayis University, Samsun, Turkey.

- Corresponding author: Dr. K. Devrim Isci Ondokuz Mayis University, Faculty of Dentistry, Department of Orthodontics, Kurupelit/Samsun, Turkey. Phone: +903623121919

Fax: +90362 4576032

E-mail: devrimiscidyahoo.com ital anomalies. ${ }^{1}$ The incidence of the disease is reported to be five to seven cases per million births in Europe ${ }^{2}$ and 4-5 per million live births in the UK and the Netherlands ${ }^{3}$ with an equal sex ratio. ${ }^{4}$ It was first recognized in 1938, 5,6 but an exact pathophysiology of the disease has not been described.

Although a majority of cases are sporadic, autosomal dominance and recessive patterns of inheritance are also reported in $10 \%$ to $20 \%$ of patients. ${ }^{17,8}$ Heterozygous mutations of the geneencoding ribosomal protein $\mathrm{S} 19$ on chromosome 19 q13.2 are detected in $25 \%$ of patients.., 10

The main clinical symptom is anemia. This is often present at birth, and in any event appears in the first year of life in more than $90 \%$ of patients. ${ }^{4,11}$ Other hematologic features of DBA are normocellular marrow with a specific deficiency of red cell 
precursors, normochromic macrocytic anemia, reticulocytopenia, normal or slightly decreased leukocyte counts, and normal or decreased platelet counts. ${ }^{1,3-6}$

Cleft lip and palate have been reported in $3-10 \%$ of DBA cases. ${ }^{7,12}$ Orofacial clefts seen in DBA are associated with non-RPS 19 mutations. ${ }^{6,13}$ It is still not known whether there is a correlation between the microtia-cleft palate phenotype and a specific DBA genotype. ${ }^{12}$ Some commonly reported characteristics of patients are extremely blonde, almost white hair, a snub noise, wide-set eyes, a thick upper lip, almond-shaped eyes, a small head, and a pointed chin. ${ }^{11}$

DBA is associated with a high incidence of malignancy. Most of the reported malignancies are acute myeloid leukemia (AML). ${ }^{6}$ Multiple combinations of therapy are used for children with DBA. Therapeutic approaches include blood transfusion, corticosteroids, iron chelating therapy, interleukin therapy, and bone marrow transplantation. ${ }^{1,3-6}$ More than $50 \%$ of patients are responsive to steroids. ${ }^{11}$ Chronic red cell transfusions in combination with iron chelating therapy or allogenic bone marrow transplantation (BMT) from an HLAidentical sibling are the only treatment options for steroid-resistant patients. ${ }^{14}$ Long-term follow-up is necessary during the course of the disease.

There is a lack of information in the literature about the oral and dental findings related to DBA. The management and unique dental findings of a 15 -year-old patient with congenital hypoplastic anemia were described in $1984 .{ }^{15}$

We present a detailed report of the periodontal, dental, and orthodontic appearances of two children with DBA.

\section{CASE REPORTS}

Case 1

A 13-year-old Caucasian girl was referred to the Dental Faculty of Ondokuz Mayis University for preoperative dental prophylaxis before organized bone marrow transplantation and to prevent any secondary inflammation postoperatively. She was the first child of a non-consanguineous parent. The other siblings of the family were healthy. There were prenatal cardiac and feeding problems due to cleft palate, which was surgically repaired when she was one year old and repeated one year later. The family reported that the cleft palate was also present in two nephews of our patient.

She had the characteristic physical properties of DBA. On physical examination, we noted her short stature and some skeletal abnormalities: hypoplastic thumbs with medial deviations (Figure 1), bilateral thenar and hypothenar atrophy, short arms, and a hemangioma on the left cheek about $1 \times 1 \mathrm{~cm}$ in diameter (Figure 2).

On admission, her hemoglobin concentration was $9.5 \mathrm{~g} / \mathrm{dl}$; mean corpuscular volume was $86 \mathrm{fL}$; and platelet and white blood cell counts were normal. She was using her daily systemic prednisolone $1 \mathrm{mg} / \mathrm{kg}$ and given erythrocyte suspension transfusions monthly.

Scars of the cleft palate and malocclusion of the whole mouth were observed during a detailed intraoral examination. She had a narrow maxilla and circular cross-bites in the maxillary arch (Figures 3 and 4).

The patient received panoramic, postero-anterior, and lateral cephalometric radiographs with detailed intraoral radiographs (Figure 5). The radiographs revealed deep dentin caries of the left mandibular first molar tooth and left maxillary first molar tooth as well as impacted mandibular third molar teeth. Her right maxillary second premolar, left first premolar, left mandibular second premolar, and right first molar teeth were missing. Her dental condition is also summarized in Table 1.

Periodontal health was qualified by gingival plaque indices and periodontal pocket depths. ${ }^{16,17} \mathrm{~A}$ periodontal WHO probe (Leibinger, Germany) was used to assess the inflammation (gingival score; scale: $0=$ none to $3=$ severe) and dental plaque accumulation (dental plaque score; scale: $0=$ none to $3=a b u n d a n t$ ). The means of the 4 regional gingival and dental plaque scores were calculated for each tooth, and the gingival and dental plaque indices in patient was calculated as the mean scores of all teeth. Pocket depth, defined as the distance between the base of the pocket and the gingival margin, was measured around each tooth, and the mean depth was calculated. Periodontal pockets, which result from destruction of the underlying periodontal tissues, measure 2 to $3 \mathrm{~mm}$ in periodontally healthy individuals. In this patient, deep periodontal pocket depths due to cleft palate at the maxillary anterior site were recorded. The test results showed high plaque accumulation. The 
other periodontal conditions were within normal limitations (Table 2).

An examination revealed skeletal Class III malocclusion because of the growth retardation of the maxilla followed by cleft palate formation. This retardation is shown by a sella-nasion anterior nasal spina (SNA) angle, which is used to determine the maxillary position to the cranium. In this case, the decrease amount of 10 revealed the backward of the maxilla when compared with the cranium. This type of skeletal Class III malocclusion is called micrognathie superior. Orthodontic measurements

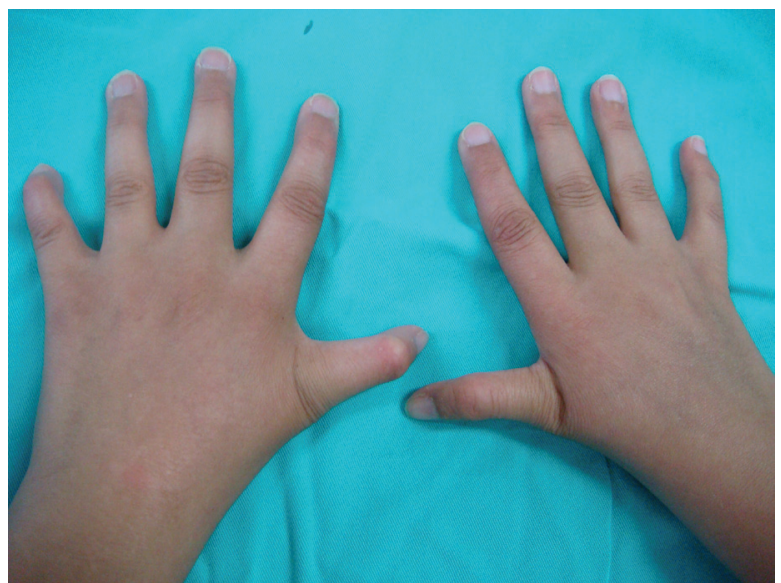

Figure 1. Hypoplasic thumbs of patient no 1, with medial deviations.

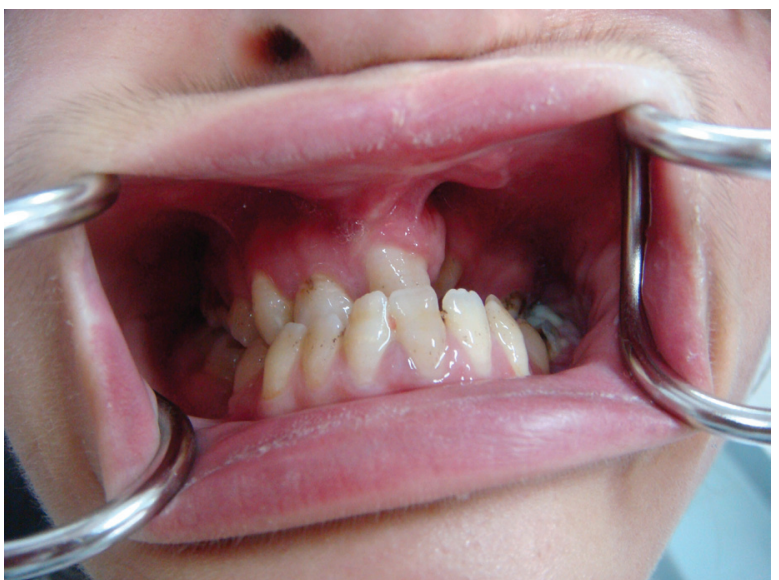

Figure 3. Malocclusion of the whole mouth of the first patient.

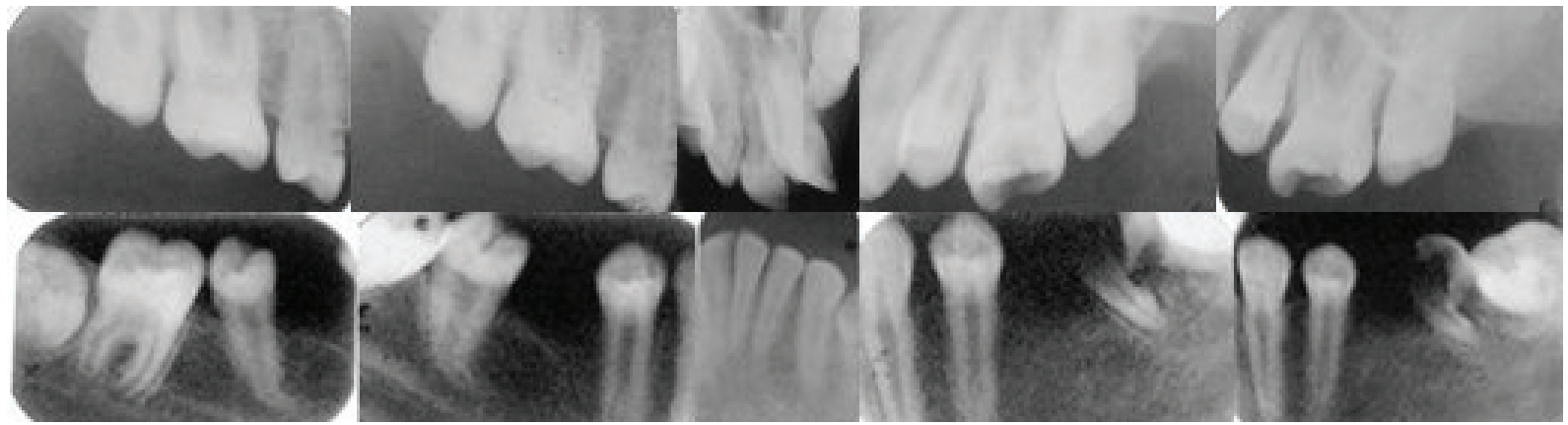

Figure 5. Detailed intraoral radiographs of patient no. 1. are shown in a lateral cephalometric radiograph in Figure 6.

After consultation with the patient's hematologist, an oral prophylaxis and topical fluoride treatment were performed. Further treatments were postponed until after the bone marrow transplantation.

\section{Case 2}

A 3-year-old Caucasian girl came to the Periodontology Department, Samsun, Turkey, for a consultation regarding her general dental health

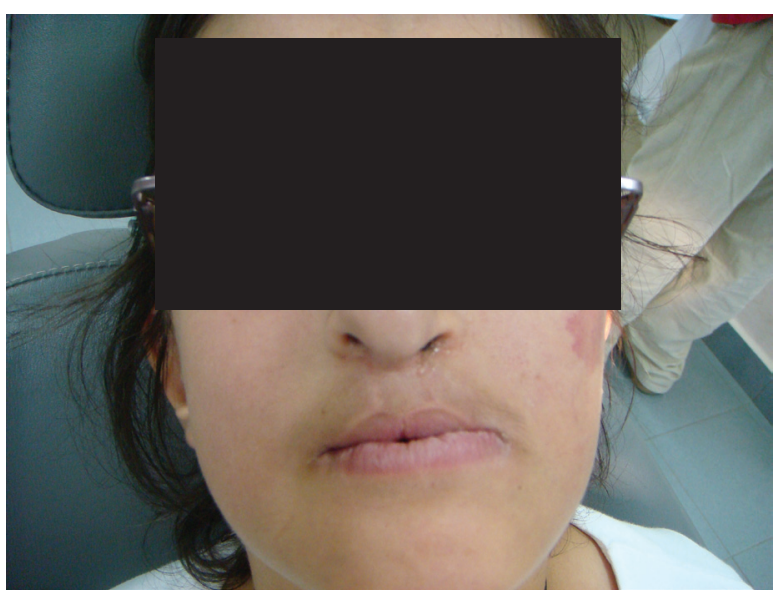

Figure 2. Hemangiom of the left cheek about $1 \times 1 \mathrm{~cm}$ in diameter of the first patient.

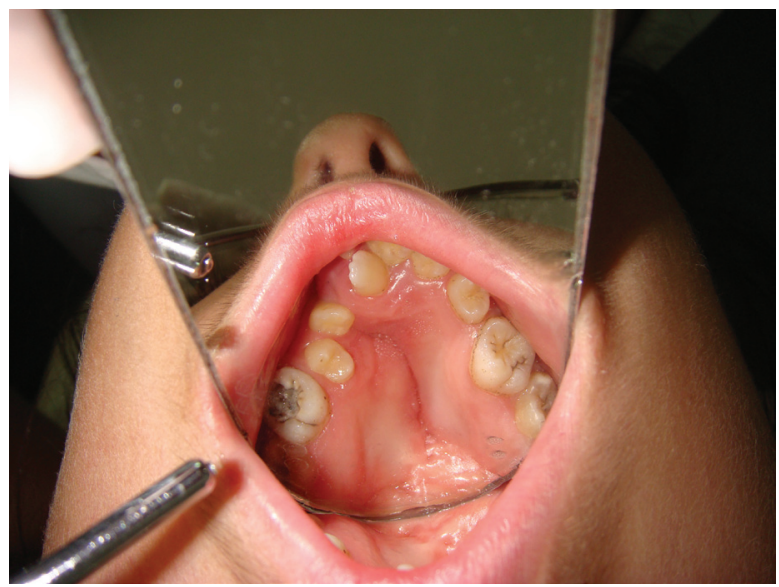

Figure 4. Scars of the cleft palate including the narrow maxilla of the patient no 1.

European Journal of Dentistry 
and an investigation of possible anomalies associated with DBA. She was the second sibling of nonconsanguineous parents. The first sibling was healthy systematically and hematologically. The patient seemed healthy, and her physical examination showed no significant physical abnormality other than a slightly pale skin. She received a daily dosage of deltacortil $(0.5 \mathrm{mg} / \mathrm{kg} /$ day) because of anemia.

An intraoral examination revealed a normal dental appearance, healthy periodontal tissues, and no detectable dental caries. Her primary teeth had all erupted and were orthodontically arranged in the right places in both the maxilla and mandible. A general physician who was consulted confirmed that routine laboratory parameters were within normal limits (hemaglobulin $11.7 \mathrm{~g} / \mathrm{dl}$ ).

Following careful intraoral and extraoral examinations, the family was informed about the importance of oral hygiene care. Instructions were given for correct tooth brushing, and the patient was asked to appear for a follow-up examination.

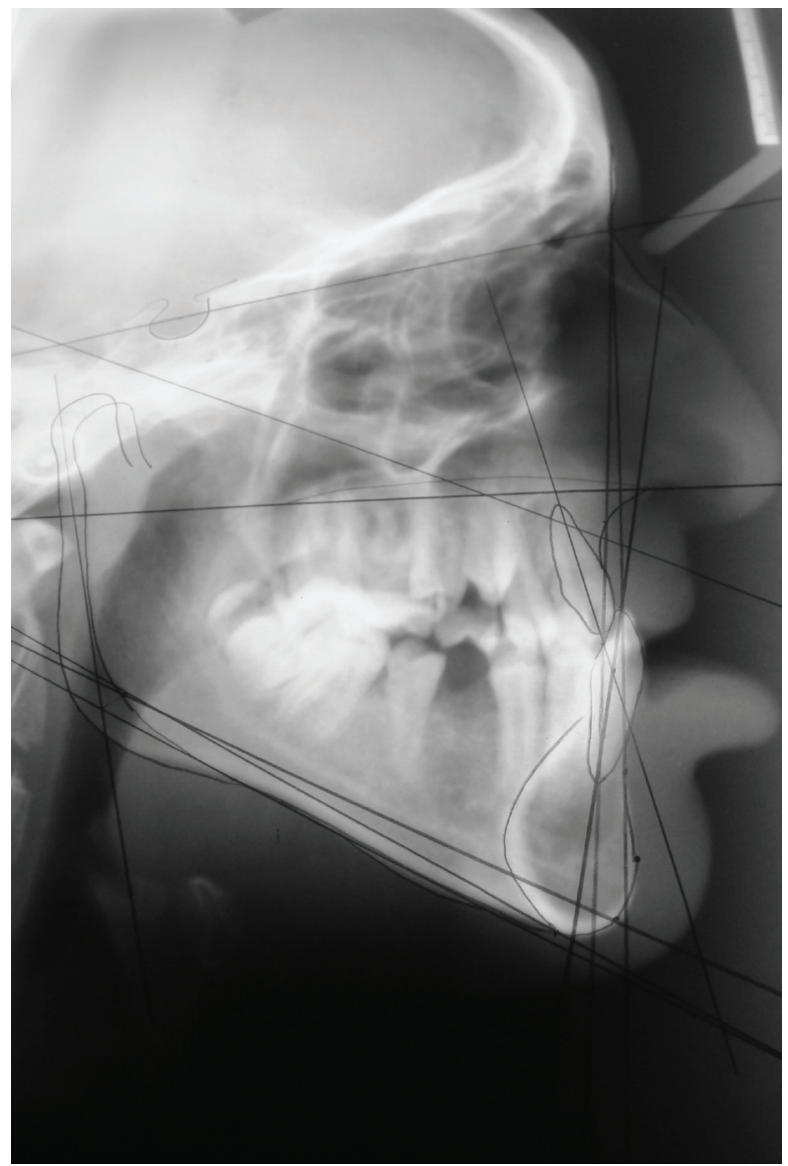

Figure 6. Orthodontic measurements related with the first patient were shown by lateral cephalometric radiograph (SNA $\left.=72^{\circ}, \mathrm{SNB}=74^{\circ}, \mathrm{ANB}=-2^{\circ}, \mathrm{SN} / \mathrm{Go}-\mathrm{Gn}=38^{\circ}\right)$.

\section{DISCUSSION}

Congenital hypoplastic anemia is a rare disease characterized by macrocytic anemia, occasional neutropenia or thrombocytosis, and deficiency of erythroblasts in the marrow. ${ }^{18}$

DBA is usually revealed in infancy, and cases have been detected before the age of 6 months. ${ }^{1}$ An exact underlying factor is not clear, and most children with this disease have long-term survival while spontaneous remission may be observed. ${ }^{15}$

Freedman ${ }^{4}$ stated phenotypic abnormalities belong to the following categories: a) craniofacial dysmorphism, including hypertelorism, microcephaly, microphtalmos, congenital cataracts or glaucoma, strabismus, microretrognathism, and a high-arched palate or cleft palate; b) prenatal or postnatal growth failure; c) neck anomalies; d) thumb malformations such as bifid thumb, duplication, subluxation, hypoplasia, or absence of the thumb.

The disease is usually treated successfully with corticosteroid therapy; however, patients who become refractory to corticosteroids are usually transfusion dependent.

Craniofacial abnormalities have been described. Gripp et $\mathrm{al}^{7}$ reported bilateral microtia, midfacial hypoplasia, cleft palate, downslating palpebral fissures, and micrognathia in two cousins with DBA. In our first case, cleft palate was reported in the patient's first cousin who did not suffer from DBA. Therefore, her malformation may not be a symptom of DBA. In our second case, none of the family members had craniofacial malformations or a medical history consistent with anemia. In the literature, craniofacial malformations, including cleft palate, bilateral microtia, downslating palpebral fissures and micrognathia, and the hematological findings of DBA were associated with Treacher-Collins syndrome, which was discussed as a distinct syndrome. ${ }^{19}$ Although both of the conditions share similarities, DBA can be differentiated based on the hematological abnormalities, the absence of a lower lid coloboma, and the absence of TCOF1 mutations. ${ }^{7}$

DBA can occur without craniofacial malformations. No examined cases of DBA with cleft palate and auditory malformations have been reported to harbor RPS ${ }^{19}$ or TCOF ${ }^{1}$ mutations. ${ }^{7}$ This genetic mutation may explain the facial differences in our patients. But it is still not clear whether there is a 
correlation between the microtia and cleft palate phenotype and a specific DBA genotype.

There is a lack of information in the literature about the oral and dental findings related to DBA. The oral and dental findings reported were severe gingivitis, multiple carious lesions, poor healing of extracted tooth sites, ${ }^{20}$ supernumerary tooth, impacted third molars, and nearly total obliteration of the coronal pulp chambers of the erupted dentition. ${ }^{15}$

Our results for patient one showed high plaque accumulation due to the patient's poor oral hygiene compliance and malformations hardened tooth brushing and reach of the patient to the whole mouth. The other periodontal conditions were within normal limitations. However, taking the age of the patient into account, increasing destruction would be a foregone conclusion for her. A careful follow-up and preventive therapies should not be missed in these kinds of patients.

Reports in the literature describing oral findings associated with DBA have not included details regarding periodontal status or missing and impacted teeth. Therefore, our study is the first to report the gingival status of the patients and a complete investigation of any orthodontic or dental abnormalities. This condition was seen only in our first case, and it is possible to associate it with the congenital cleft palate of that patient.

\section{REFERENCES}

1. Costa L, Willig TN, Fixler J, et al. Diamond-Blackfan Anemia. Curr Opin Pediatr 2001;13:10-15.

2. Ball SE, McGuckin CP, Jenkins G, et al. Diamond-Blackfan anaemia in the United Kingdom: analysis of 80 cases from a 20-year birth cohort. Br J Haemotol 1996;94:615-653.

3. Krijanovski OI, Sieff CA. Diamond-Blackfan anemia. Hematol Oncol Clin North Am 1997;11:1061-1077.

4. Freedman MH. Diamond-Blackfan anaemia. Bailliere's Clinical Haemotology 2000;13:391-406.

5. Diamond LK, Blackfan KD. Hypoplastic anemia. Am J Dis Child 1938;56:464-467.

6. Vlachos A, Klein GW, Lipton JM. The Diamond Blackfan anemia registry: Tool for investigating the epidemiology and biology of Diamond-Blackfan Anemia. J Pediatr Hematol Oncol 2001;23:377-382.

7. Gripp KW, McDonald-McGinn DM, Rossa DL, et al. Bilateral microtia and cleft palate in cousins with Diamond-Blackfan anemia. Am J Med Genet 2001;101:268-274.
8. Orfali KA, Ohene-Abukawa Y, Ball SE. Diamond-Blackfan anemia in UK: Clinical and genetic heterogenecity. $\mathrm{Br} \mathrm{J}$ Haematol 2004;125:243-252.

9. Draptchinskaia N, Gustavsson P, Andersson B, et al. The gene encoding ribosomal protein S19 is mutated in Diamond-Blackfan anaemia. Nat Genet 1999;21:169-175.

10. Willig TN, Draptchinskaia N, Dianzani I, et al. Mutations in ribosomal protein $\mathrm{S} 19$ gene and Diamond Blackfan anemia; wide variations in phenotypic expression. Blood 1999;94:4294-4306.

11. Halperin DS, Freedman MH. Diamond-Blackfan anemia: etiology, pathophysiology, and treatment. Am J Pediatr Hematol Oncol 1989;11:380-394.

12. Lipton JM, Atsidaftos E, Zyskind I, et al. Improving clinical care and elucidating the pathophysiology of Diamond Blackfan anemia: An update from the Diamond Blackfan Anemia Registry. Pediatr Blood Cancer 2006;46:558-564.

13. Vlachos A, Klein GW, Avarello JT, et al. Diamond Blackfan anemia (DBA) and associated orofacial clefts (OFC): A Diamond Blackfan Anemia Registry (DBAR) analysis. J Pediatr Hematol Oncol 2002;24:1426.

14. Mushigima H, Gale RP, Rowlings PA, et al. Bone marrow transplantation for Diamond-Blackfan anemia. Bone Marrow Transplant 1995; 15:55-58.

15. Willis TB, Seale NS. Oral manifestations in congenital hypoplastic anemia (Diamond-Blackfan anemia): a clinical report. Pediatr Dent 1984;6:162-163.

16. Löe H, Silness J. Periodontal disease in pregnancy. I. Prevalence and severity. Acta Odontol Scand 1963;21:533-551.

17. Silness J, Löe H. Periodontal disease in pregnancy. II. Correlation between oral hygiene and periodontal condition. Acta Odontol Scand 1964;22:121-135.

18. Gazda HT, Sieff CA. Recent insights into the pathogenesis of Diamond-Blackfan anaemia. Br J Haematol 2006;135:149157.

19. Hasan R, Inoue, S. Diamond Blackfan anemia associated with Treacher Collins syndrome. Pediatr Hematol Oncol 1993;10:261-265.

20. Sanger RG, Wilson GA. Congenital hypoplastic anemia (Diamond-Blackfan syndrome). J Oral Med 1982;37:8-13. 